\title{
A Simple Model to Explain Expensive Index Call Options
}

\author{
Sang Baum Kang \\ Stuart School of Business, Illinois Institute of Technology, Chicago, IL, USA \\ Email: skang21@stuart.iit.edu
}

How to cite this paper: Kang, S.B. (2017) A Simple Model to Explain Expensive Index Call Options. Theoretical Economics Letters, 7, 316-323.

https://doi.org/10.4236/tel.2017.73024

Received: February 7, 2017

Accepted: March 13, 2017

Published: March 16, 2017

Copyright (C) 2017 by author and Scientific Research Publishing Inc. This work is licensed under the Creative Commons Attribution International License (CC BY 4.0).

http://creativecommons.org/licenses/by/4.0/

\begin{abstract}
According to the empirical finance literature, S\&P 500 Index call options frequently violate the stochastic dominance upper bounds. In other words, index call options in the US are frequently overpriced. I propose a theoretical model to explain the reason for this. A simple economic model in this article reveals that when agents are sufficiently heterogeneous, a call option may be overpriced from the perspective of the representative agent. The key economic intuitions can be summarized as follows: First, if agents are sufficiently heterogeneous, a bullish agent, who is hungry for the "exposure" to a stock, may buy an expensive call option from a constrained bearish agent. Second, even though a call option is fairly priced from the perspectives of heterogeneous market participants, it may be overpriced from the perspective of the representative agent. Assuming reasonable parameters of heterogeneity, I find that a call option price violates the representative agent's stochastic dominance upper bound.
\end{abstract}

\section{Keywords}

Heterogeneity in Beliefs, Stochastic Dominance Upper Bound, Index Option, Call Option, Representative Agent

\section{Introduction}

Index option prices tend to be greater than theoretical asset pricing models predict (see, e.g., [1] among many others). Such expensive index options are one of the unsolved puzzles in the finance literature. However, solid understanding of the expensive index options is academically and practically important, because index options are one of the most liquid derivative securities in the United States and various economic agents use index options for speculation, hedging, and arbitrage purposes.

It is well known that S\&P 500 Index put option premiums are greater than 
classical asset pricing models predict (see, e.g., [1] and [2]). When it comes to index call options ${ }^{1}$, [3] and [4] reported that S\&P 500 Index call options are so expensive that stochastic dominance (hereafter, "SD") trading opportunities arise. Specifically, index call options frequently violate the SD upper bounds, and such violations are not consistent with market equilibrium, because they give rise to preference-free "good-sell opportunities". However, it is difficult to find a paper providing a theoretical explanation for why such "good-sell opportunities" are so common. To fill this gap, I theoretically investigate whether heterogeneity in investors' beliefs may provide an explanation.

In this paper, I present a simple economic model to show that a sufficiently large amount of belief dispersion increases a call option price up to the point that it violates the representative agent's SD upper bound. This paper considers an economy with a single consumption good and a single risky underlying asset, which yields an uncertain number of consumption goods. Agents there may have heterogeneity in beliefs on expected output. These heterogeneous agents trade a stock and an option on the stock to balance their expected portfolio gain and risk. In the presence of sufficiently large belief dispersion, a bullish agent whose stock position limit is effectively binding may buy a call option to gain more exposure to the stock ${ }^{2}$. When the heterogeneity in beliefs is even more pronounced, the bullish agent ends up with buying an overpriced call from the constrained bearish agent because a purchase of the expensive call option increases her expected utility. Even though such a call option is fairly priced to heterogeneous agents in the model economy, it is overpriced from the perspective of the representative agent.

The contribution of this paper is to show why preference-free "good-sell opportunities" of call options, as documented in [3], arise in equilibrium. Assuming reasonable parameters of heterogeneity, I find that a call option price violates the representative agent's stochastic dominance upper bound. By providing a novel economic model, this paper sheds light on understanding of why index call options tend to be expensive. Specifically, the theoretical model in this paper suggests that index call options are expensive because investors' beliefs are heterogeneous.

The remainder of this paper is structured as follows. Section 2 describes economic environment. Section 3 presents the model economy and its equilibria. Section 4 is the conclusion.

\section{Economic Environment}

Consider a single consumption good and one underlying asset. Investment into one share of the underlying risky asset returns an uncertain number $\tilde{S}$ of consumption goods at the end of the period. A gross interest rate $R_{f}>1$ is ex${ }^{1} \mathrm{~A}$ call option is the right but not the obligation to buy an underlying asset at a predetermined price on a pre-specified date.

${ }^{2}$ If the bearish agent, who sells the stock to the bullish agent, has a binding lower position limit, the upper position limit of the bullish agent is effectively binding even though the bullish agent does not have any upper position limit. 
ogenous and non-stochastic. The investment opportunities set also includes a call option on $\tilde{S}$ with strike $K$ which pays

$$
\tilde{c} \equiv \max [\tilde{S}-K, 0] \text {. }
$$

This economy is populated by $I$ agents indexed by $1, \cdots, I$, who trade their shares of $\tilde{S}$ and the call option at the beginning of the period. The net supply of the stock (a share of the risky asset) is $V$ and the net supply of call options is zero.

The agents are heterogeneous in their beliefs on output of the risky asset. Specifically, agent $i$ believes that

$$
\tilde{S}=\hat{S}_{i}+\hat{\sigma}_{i} Z
$$

where $\hat{S}_{i}$ and $\hat{\sigma}_{i}$ are agent $i$ s belief on expected output and the standard deviation of output; $Z$ is a standard normal random variable. The constant absolute risk aversion (hereafter, "CARA") of agent $i$ is $\gamma_{i}$, i.e., agent $i$ s utility function is $u_{i}(W)=-\exp \left(-\gamma_{i} W\right)^{3}$.

All agents have an equal endowment of stock at the beginning of the period, namely, $(V / I)$ shares of stock. After trading, agent $i$ holds $\varepsilon_{i}^{S}$ shares of stocks and $\varepsilon_{i}^{c}$ shares of calls; a negative $\varepsilon_{i}^{S}$ or $\varepsilon_{i}^{c}$ means a short position. For example, if agent $i$ buys one stock and sells two calls, $\varepsilon_{i}^{S}=(V / I)+1$ and $\varepsilon_{i}^{c}=-2$; if agent $i$ does not buy any stock or call, $\varepsilon_{i}^{S}=(V / I)$ and $\varepsilon_{i}^{c}=0$. Let $S(0)$ and $c(0)$ be the market price of stock and call, respectively. Agent is portfolio at the beginning of the period consists of $\left(\left((V / I)-\varepsilon_{i}^{S}\right) S(0)-\varepsilon_{i}^{c} c(0)\right)$ consumption goods of the risk-free asset, $\varepsilon_{i}^{S}$ shares of stock, and $\varepsilon_{i}^{c}$ shares of call. Her wealth at the end of the period is

$$
\tilde{W}_{i}=\left\{\left((V / I)-\varepsilon_{i}^{S}\right) S(0)-\varepsilon_{i}^{c} c(0)\right\} R_{f}+\varepsilon_{i}^{S} \tilde{S}+\varepsilon_{i}^{c} \tilde{c} .
$$

Agent $i$ may have position limits, e.g., $\varepsilon_{i}^{S} \geq 0$ and $\varepsilon_{i}^{c} \geq-1$. To determine her optimal $\varepsilon_{i}^{S}$ and $\varepsilon_{i}^{c}$ given $S(0)$ and $c(0)$, agent $i$ solves a (constrained) maximization problem of her expected utility. Using properties of normal and log-normal distribution and the change of variables, I have

$$
E_{i}\left[u_{i}\left[\tilde{W}_{i}\right]\right]=u_{i}[A] \times \Phi\left[z_{i}^{c}+\gamma_{i} \varepsilon_{i}^{S} \hat{\sigma}_{i}\right]+u_{i}[B] \times\left(1-\Phi\left[z_{i}^{c}+\gamma_{i}\left(\varepsilon_{i}^{S}+\varepsilon_{i}^{c}\right) \hat{\sigma}_{i}\right]\right)
$$

where

$$
\begin{gathered}
A \equiv\left\{\left((V / I)-\varepsilon_{i}^{S}\right) S(0)-\varepsilon_{i}^{c} c(0)\right\} R_{f}+\varepsilon_{i}^{S} \hat{S}_{i}-\left(\gamma_{i} / 2\right)\left(\varepsilon_{i}^{S}\right)^{2}\left(\hat{\sigma}_{i}\right)^{2} ; \\
B \equiv\left\{\left((V / I)-\varepsilon_{i}^{S}\right) S(0)-\varepsilon_{i}^{c} c(0)\right\} R_{f}+\left(\varepsilon_{i}^{S}+\varepsilon_{i}^{c}\right) \hat{S}_{i}-\left(\gamma_{i} / 2\right)\left(\varepsilon_{i}^{S}+\varepsilon_{i}^{c}\right)^{2}\left(\hat{\sigma}_{i}\right)^{2}-\varepsilon_{i}^{c} K ;
\end{gathered}
$$

$u_{i}(\cdot)$ is her utility; $E_{i}[\cdot]$ is the expectation under her subjective measure; $\Phi[\cdot]$ is the standard normal c.d.f.; and $z_{i}^{c} \equiv\left(K-\hat{S}_{i}\right) / \hat{\sigma}_{i}$. Agent $i$ maximizes Equation (2) by taking optimal positions of $\varepsilon_{i}^{S}$ and $\varepsilon_{i}^{c}$.

\subsection{Equilibrium under Homogeneity in Beliefs}

Homogeneity in preference and beliefs implies

${ }^{3}$ The constant absolute risk aversion preference is used for analytic tractability. 


$$
\gamma_{i}=\gamma, \hat{S}_{i}=\hat{S}, \hat{\sigma}_{i}=\hat{\sigma}, \varepsilon_{i}^{S}=(V / I) \text {, and } \varepsilon_{i}^{c}=0 \text { for all } i=1, \cdots, I \text {. }
$$

Substituting Equation (3) into a first order condition (hereafter, "FOC") of Equation (2), I obtain the stock price:

$$
S(0)=\left\{\hat{S}-\gamma(\hat{\sigma})^{2}(V / I)\right\} / R_{f} .
$$

Similarly, the call price is:

$$
c(0)=\frac{\left(\hat{S}-\gamma(\hat{\sigma})^{2}(V / I)-K\right)\left(1-\Phi\left[z^{c}+\gamma \hat{\sigma}(V / I)\right]\right)+\phi\left[z^{c}+\gamma \hat{\sigma}(V / I)\right] \hat{\sigma}}{R_{f}}
$$

where $z^{c}=(K-\hat{S}) / \hat{\sigma}$ and $\phi[\cdot]$ is the standard normal p.d.f.

\subsection{Equilibrium under Heterogeneity in Beliefs}

I numerically calculate equilibrium prices, $S(0)$ and $c(0)$, and volumes, $\varepsilon_{1}^{S}, \cdots, \varepsilon_{I}^{S}$, and $\varepsilon_{1}^{c}, \cdots, \varepsilon_{I}^{c}$, which maximize Equation (2) and satisfy two market clearing conditions:

$$
\begin{gathered}
\varepsilon_{1}^{S}+\cdots+\varepsilon_{I}^{S}=V ; \text { and } \\
\varepsilon_{1}^{c}+\ldots+\varepsilon_{I}^{c}=0 .
\end{gathered}
$$

The algorithm can be summarized as follows:

1) Pick reasonable initial candidates of equilibrium $S(0)$ and $c(0)$ such as Equations ((4) and (5)).

2) Calculate each agent $i$ s optimal $\varepsilon_{i}^{S}$ and $\varepsilon_{i}^{c}$ by maximizing Equation (2). Sum optimal $\varepsilon_{i}^{S}$ and $\varepsilon_{i}^{c}$ over all $i=1, \cdots, I$ into $\sum_{i=1}^{I} \varepsilon_{i}^{S}$ and $\sum_{i=1}^{I} \varepsilon_{i}^{c}$.

3) If $\sum_{i=1}^{I} \varepsilon_{i}^{s} \cong V$ and $\sum_{i=1}^{I} \varepsilon_{i}^{c} \cong 0$, stop the algorithm.

4) If $\sum_{i=1}^{I} \varepsilon_{i}^{S}$ is greater (less) than $V$, increase (decrease) $S(0)$. If $\sum_{i=1}^{I} \varepsilon_{i}^{c}$ is greater (less) than 0 , increase (decrease) $c(0)$. Go to step 2.

\subsection{The Representative Agent}

The representative agent, or the social planner, has preference of

$$
u_{m}(W)=-\exp \left[-\gamma_{m} W\right]
$$

where $\gamma_{m}$ is given by

$$
\gamma_{m} \equiv 1 / \tau_{m}, \tau_{m} \equiv \sum_{i=1}^{I} \tau_{i}, \text { and } \tau_{i} \equiv 1 / \gamma_{i}
$$

and believes that output of underlying asset is

$$
\tilde{S}=\hat{S}_{m}+\hat{\sigma}_{m} Z
$$

where $\left(\hat{\sigma}_{m}\right)^{2}$ is given by the harmonic average of $\left(\hat{\sigma}_{i}\right)^{2}$ s weighted by $\tau_{i}$ s, i.e.,

$$
\left(\hat{\sigma}_{m}\right)^{2}=1 /\left\{\sum_{i=1}^{I} \frac{\tau_{i}}{\tau_{m}} \frac{1}{\left(\hat{\sigma}_{i}\right)^{2}}\right\}
$$


and $\hat{S}_{m}$ is the average of $\hat{S}_{i}$ s weighted by $\tau_{i} /\left(\hat{\sigma}_{i}\right)^{2}$, i.e.,

$$
\hat{S}_{m}=\left\{\sum_{i=1}^{I}\left(\tau_{i} /\left(\hat{\sigma}_{i}\right)^{2}\right) \hat{S}_{i}\right\} /\left\{\sum_{i=1}^{I}\left(\tau_{i} /\left(\hat{\sigma}_{i}\right)^{2}\right)\right\},
$$

at competitive equilibrium. (See Appendix A for proof.)

\subsection{Stochastic Dominance upper Bound}

The representative agent's SD upper bound is

$$
c_{m}^{U B}(0)=\left(S(0) / \hat{S}_{m}\right)\left\{\left(\hat{S}_{m}-K\right)\left(1-\Phi\left[z_{m}^{c}\right]\right)+\phi\left[z_{m}^{c}\right] \hat{\sigma}_{m}\right\}
$$

where $z_{m}^{c} \equiv\left(K-\hat{S}_{m}\right) / \hat{\sigma}_{m}$, as I prove in Appendix A.

\section{The Model Economy}

In this model economy populated by two agents in the presence of heterogeneity in beliefs and position limits, I calculate equilibrium stock and call prices, and compare them with the representative agent's SD upper bound to show that sufficiently large heterogeneity may lead to a violation of the representative agent's SD upper bound.

\subsection{Description}

For simplicity, consider two agents, $\mathrm{A}$ and $\mathrm{B}$, who have heterogeneous beliefs on the expected output: $\hat{S}_{A}=10+H_{\mu}$ and $\hat{S}_{B}=10-H_{\mu}$ where $H_{\mu}$ is a parameter for heterogeneity. For example, $H_{\mu}=0$ means that the two agents are homogeneous in beliefs on $\hat{S}_{i}$. In contrast, if $H_{\mu}>0, \mathrm{~A}$ and B are heterogeneous in their beliefs on the expected output. Each agent is endowed with +1 stock. Stock short-sell limits are imposed, and each agent can supply the call option up to amount $-1^{4}$.

In equilibrium, the stock and call prices and volumes are numerically determined so that Equations ((6) and (7)) are satisfied. On the other hand, the representative agent's preference and her belief in the expected output are determined by Equations ((9), (11), and (12)), and she prices the stock and call option accordingly.

\subsection{Numerical Results}

Figure 1 summarizes the numerical results. When $H_{\mu}=0$ (i.e. agents are homogeneous in beliefs), the agents do not trade any stock (call option), as one can observe in the first plot of the first (second) row. This is logical because the second plot in the first (second) row shows that the two agents have the same subjective pricing of the stock (call option), which is identical to the equilibrium market price. As the third plot in the first (second) row illustrates, the market equilibrium stock (call) price is also the same as the representative agent's pricing.

${ }^{4}$ Even though some amount of short-selling is allowed, the main results do not change. I chose call supply limit of -1 to ensure that the call options outstanding do not exceed the total endowment of stock. 

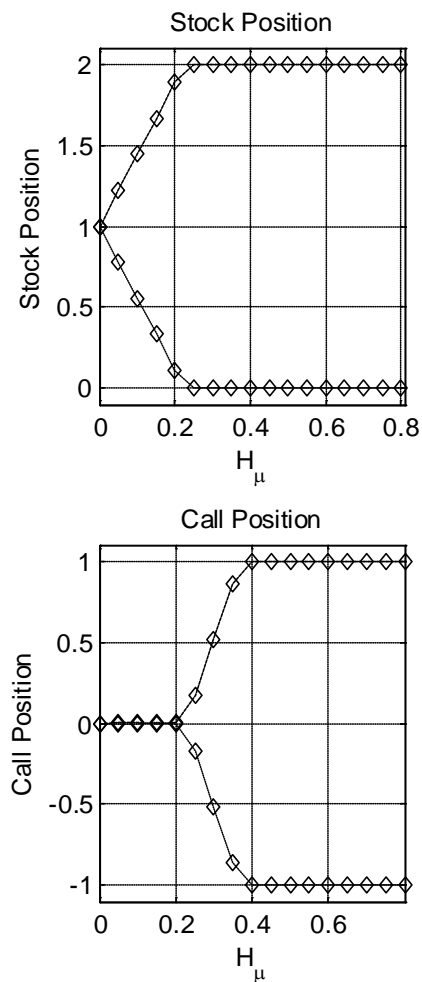

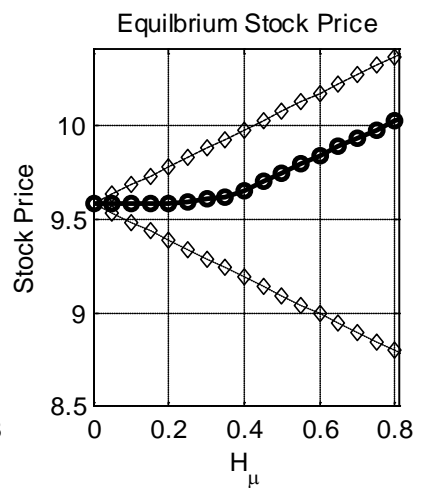

Equilibrium Call Price

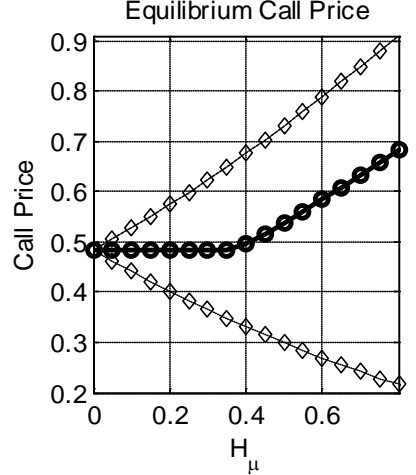

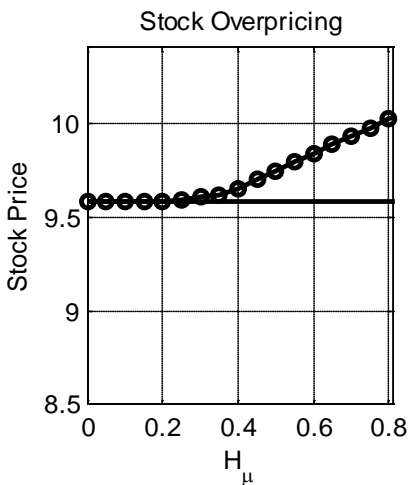

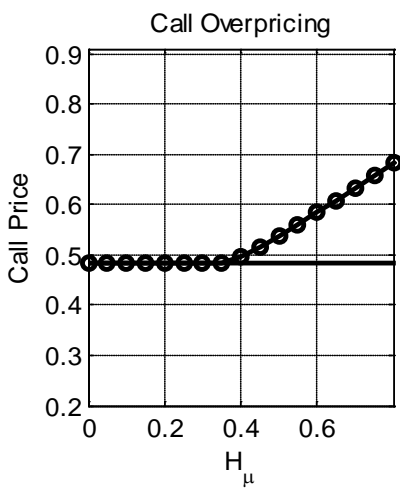

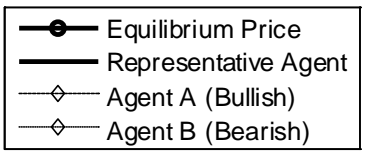

SD Upper Bound Violation

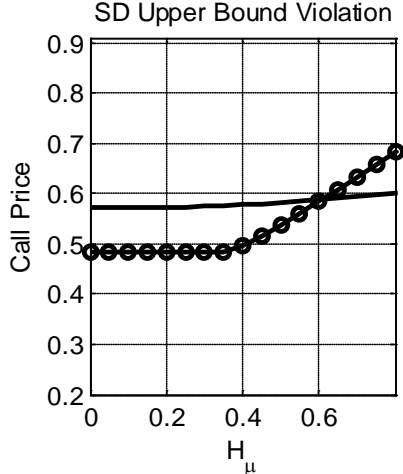

Figure 1. Stock and call volumes and prices under various $H_{\mu}$ s. $\hat{S}_{A}=10+H_{\mu}, \hat{S}_{B}=10-H_{\mu}$ and $\hat{\sigma}_{i}=1.5$ are assumed. Each agent's endowment for the stock is +1 ; stock short-sell constraints and call option lower position limits of -1 are imposed. Furthermore, this figure uses $\mathrm{K}=10, \mathrm{~T}=1, \mathrm{r}=0.02$, and $\gamma_{i}=0.1$. The $1^{\text {st }}$ column depicts stock and call positions of each agent (i.e., $\varepsilon_{i}^{S}$ and $\varepsilon_{i}^{c}$. The $2^{\text {nd }}$ column compares the equilibrium stock and call prices with each agent's subjective pricing calculated by (4) and (5) against agent i's subjective belief. The $3^{\text {rd }}$ column compares the equilibrium stock and call prices with the representative agent's pricing (i.e., (4) and (5) against the representative agent's belief). Finally, the $4^{\text {th }}$ column compares call prices with the SD upper bound of the representative agent calculated by (13).

When $H_{\mu}>0$ (i.e. agents are heterogeneous in beliefs), bullish agent A buys the stock from bearish agent $\mathrm{B}$, because agent $\mathrm{A}$ wants more "exposure" to the stock in order to increase her expected utility under her subjective measure, which is different from agent B's. Agent A takes the stock position so that her expected profit and variance are balanced. As $H_{\mu}$ increases, bullish agent A buys more stocks from bearish agent $\mathrm{B}$.

For $0<H_{\mu} \leq 0.2$, agents $\mathrm{A}$ and $\mathrm{B}$ trade the stock but do not trade the call option, because unless agent B's stock short-sell limit is binding, stock trading sufficiently balances the two agents' expected return and risk. The market equilibrium stock and call prices are still the same as the representative agent's pricing.

When $0.2<H_{\mu}<0.4$, agent B's stock short-sell limit is binding, and the agents trade both the stock and the call option. If bearish agent B's short-sell limit is binding, bullish agent A cannot buy any more stocks. Because agent A needs extra "exposure" to the stock, she buys an overpriced stock and further purchases the call option. Because of the friction of binding stock short-sell limit, the equilibrium stock price is greater than the representative agent's price, as in the third plot of the first row. However, because the optimal call demand does 
not reach the call position limit, there is no need for call overpricing.

In the cases of $0.4 \leq H_{\mu}<0.6$, both the stock and call position limits are binding, and both the stock and call prices are greater than the representative agent's pricing. The equilibrium stock and call prices become greater than the representative agent's pricing when the stock and call position limits are binding.

Finally, if $H_{\mu}>0.6$, the representative agent's SD upper bound is violated, as the fourth plot of the second row illustrates. Specifically, $H_{\mu}=0.65$ leads to $\hat{S}_{A}=10.65$ and $\hat{S}_{B}=9.35$; agent A (B) expects $6.5 \%$ more (less) $\hat{S}_{i}$ than the simple average $\left(\left(\hat{S}_{A}+\hat{S}_{B}\right) / 2=10\right)$. In comparison to the model parameter of $\hat{\sigma}_{i}=1.5$ (equivalent to $15 \%$ ) and S\&P 500 Index's annualized volatility (e.g., VIX historically ranges from $10 \%$ to $60 \%$ annualized.), $\pm 6.5 \%$ is reasonably small. In this model economy, relatively modest heterogeneity in beliefs leads to an SD upper bound violation of the representative agent.

\section{Conclusion}

In quest of a potential resolution of "overpriced index options puzzle," this paper finds that if agents are heterogeneous in beliefs, an equilibrium call price may be greater than the SD upper bound of the representative agent. The key economic intuitions can be summarized as follows:

1) If agents are sufficiently heterogeneous, a bullish agent, who is hungry for the "exposure" to a stock, may buy an expensive call option from a constrained bearish agent.

2) Even though a call option is fairly priced from the perspectives of heterogeneous market participants, it may be overpriced from the perspective of the representative agent.

My model uncovers that the heterogeneity in beliefs results in expensive call options. This finding has practical implications: Option writers may want to quantify and trace heterogeneity in beliefs to find "good sell" opportunities of index call options. My results are also important to regulators who are responsible for maintaining financial market stability: If investors' beliefs about the market are very heterogeneous, the call option price may be extremely high, and regulators may need to worry about the market stability.

There are drawbacks of my model: First, for analytic tractability, I use CARA preference. Second, for simplicity, I assume away market microstructure such as bid-ask spreads. Even if I use different preference and model bid-ask spreads, the main results may not change qualitatively. However, the required heterogeneity to induce the SD upper bound violation may change.

The current research opens a new avenue for future academic research. First, extending my model, a theorist may devise a next generation of model with more realistic economic setting and investigate whether s/he can find any other incremental economic intuitions. Second, an empiricist may quantify heterogeneity in beliefs, gather real-time price data, and statistically test whether my theory is consistent with the data. 


\section{References}

[1] Chambers, D.R., Foy, M., Liebner, J. and Lu, Q. (2014) Index Option Returns: Still Puzzling. Review of Financial Studies, 27, 1915-1928. https://doi.org/10.1093/rfs/hhu020

[2] Bondarenko, O. (2014) Why Are Put Options So Expensive? Quarterly Journal of Finance, 4, 1-50. https://doi.org/10.1142/S2010139214500153

[3] Constantinides, G.M., Czerwonko, M., Jackwerth, J.C. and Perrakis, S. (2011) Are Options on Index Futures Profitable for Risk Averse Investors? Empirical Evidence. Journal of Finance, 66, 1407-1437. https://doi.org/10.1111/j.1540-6261.2011.01665.x

[4] Constantinides, G.M., Jackwerth, J.C. and Perrakis, S. (2009) Mispricing of S\&P 500 Index Options. Review of Financial Studies, 22, 1247-1277. https://doi.org/10.1093/rfs/hhn009

[5] Ritchken, P.H. (1985) On Option Pricing Bounds. Journal of Finance, 40, 12191233. https://doi.org/10.1111/j.1540-6261.1985.tb02373.x

\section{Appendix A: Proofs}

Representative Agent's Preference and Belief. The social planning problem is

$$
\max \left\{-\sum_{i=1}^{I} \lambda_{i} E\left[\exp \left(-\gamma_{i} \tilde{W}_{i}\right) \tilde{Z}_{i}\right]\right\}
$$

s.t. $\sum_{i=1}^{I} \tilde{W}_{i}=\tilde{W}_{m}$ where $\lambda_{i}$ is the Lagrangian multipliers for Pereto optimum, and $\tilde{Z}_{i}$ is the Radon-Nikodyn derivative of agent is belief w.r.t. any reference belief in a normality form. Specifically, $\tilde{Z}_{i}$ is the ratio of two normal p.d.f.s. Combining FOCs, the optimal sharing rule is

$$
\tilde{W}_{i}=\left(\frac{\tau_{i}}{\tau}\right) \tilde{W}_{m}+\tau_{i}\left[\ln \left(\lambda_{i} \tilde{Z}_{i} \gamma_{i}\right)-\sum_{j=1}^{I}\left(\frac{\tau_{j}}{\tau}\right) \ln \left(\lambda_{j} \tilde{Z}_{j} \gamma_{j}\right)\right] .
$$

Substituting the optimal sharing rule into the social planning problem, the representative agent's expected utility is proportional to $-E\left[\exp \left(-\gamma_{m} \tilde{W}_{m}\right) \tilde{Z}_{m}\right]$ where $\tilde{Z}_{m} \equiv \prod_{i=1}^{I} \tilde{Z}_{i} / E\left[\prod_{i=1}^{I} \tilde{Z}_{i}\right]$. Observe that $\lambda_{i}$ does not show up in the representative agent's Radon-Nikodyn derivative $\tilde{Z}_{m}$. Organizing the terms leads to the required result.

Stochastic Dominance Upper Bound on a European Call. Because the representative agent has a positive endowment of stock and CARA preference, the monotonicity condition is satisfied. The payoff of a European call option satisfies the convexity condition. Adapting [5], I obtain

$$
\begin{aligned}
c_{m}^{U B}(0) & =\left(S(0) / \hat{S}_{m}\right) E[\max [0, \tilde{S}-K]] \\
& =\left(S(0) / \hat{S}_{m}\right)\left\{\left(\hat{S}_{m}-K\right)\left(1-\Phi\left[z_{m}^{c}\right]\right)+\phi\left[z_{m}^{c}\right] \hat{\sigma}_{m}\right\} .
\end{aligned}
$$

